\title{
Case Report Writing
}

\section{Vijaykumar Dehannathparambil Kottarathil ${ }^{1}$}

Received: 22 September 2020 / Accepted: 24 September 2020 / Published online: 2 October 2020

(C) Indian Association of Surgical Oncology 2020

Willim Osler, a Canadian Physician and one of the four founding fathers of John Hopkins Hospital in 1920, said that "Always note and record the unusual... Publish it. Place it on permanent record as a short, concise note. Such communications are always of value."

Many advances in clinical practice have occurred as a result of valuable information and new ideas presented in case reports.

Case report writing is possibly the first step in scientific writing for a health care professional. Case report writing may be regarded as the bottom rung of the hierarchy of evidence in literature; however, it is probably the best way for initiation of a young medical professional into the world of scientific writing. It requires less effort, while retaining all the essentials of basic methodology of scientific writing. It also requires fewer resources.

A case report could either be an entirely new disease entity hitherto unknown or should contribute new knowledge to the understanding, diagnosis, or management of a known disease. The important thing to remember while choosing a case for reporting is to ensure that it contains some learning point, either a new technique, a new diagnostic point, a different way of treating, or an unexpected response to conventional treatment. There should be some new knowledge gained, and the reader must be able to identify and use the learning point from the case if confronted with a similar situation. Educational value rather than extreme rarity is what an editor will look for in a case report.

Case reports are generally shorter than other articles. A definite structure is to be followed with a title, abstract, a brief introduction including literature review, a description of the actual case, followed by discussion, and conclusion. The actual format of the case report will differ according to the

Vijaykumar Dehannathparambil Kottarathil

dkvijaykumar@aims.amrita.edu

1 Amrita Centre for Breast diseases, Amrita Institute of Medical Sciences and Research Center, Kochi, Kerala 682041, India journal chosen, but most will have a similar sort of structure. There will be a limitation on the number of words, references, figures, and tables, and this should be checked before submission.

Other forms of short communication with the readers include letter to editor, case series, and images of rare disease or unusual presentation.

A letter to the editor should be written immediately after the appearance of the article concerned. If there is a delay of a few months after publication, most editors would not be interested in publishing the same. It has to be short and one needs to come directly to the point. Do not be afraid of criticizing or pointing out differences if any. Remember that the original authors will be given the right to respond to the letter and it may be published in the same or subsequent issue. Letter to editor may be in the form of an observation or comment. The former is an additional piece of information which may add to what has been written while the latter is a criticism of some aspect of the published work. Letters written to the editor or the author should contain objective and constructive interpretations or discussions on medical, scientific, or general areas of interest. They should have an objective and give a message with a brief and clear language. Do not use it to settle personal scores; Do not use it to present your own additional data which has not been peer-reviewed or published.

Please read the instruction to authors for that journal carefully before submitting. Each journal may have slightly different guidelines and restrictions. For example, in this journal, the word limit is 750 with 1 table, one figure, and 10 references. Many journals may also have restrictions on the number of authors permitted.

Images of rare disease or unusual presentation of common disease with exceptional interest is also worth publishing as this may be of interest to a lot of readers. Ensure that the image quality is good and that all identifying markers are removed. If some form of identification is inevitable, ensure that written permission is obtained from the patient.

Our medical training tends to focus more on academic aspects with a neglect of the scientific aspects. Besides this, 
work overload, absence of good mentors, low levels of enthusiasm for publishing, and restricted access to bibliographic resources make the job harder.

Making writing a case report as a part of the curriculum for all medical students during their undergraduate training, especially as a part of their clinical postings, would go a long way in initiating them into the different aspects of scientific writing. They gain experience about the editorial process, literature search, and the various problems involved in the actual process of scientific writing. The beginners can also refer to "A manual on preparing surgical case reports for presentation or publication" by Dr.Whiton S Paine published in 2003 which gives fairly detailed steps in preparing a case report.

Quality of published surgical case reports is a matter of concern. This can be overcome if one follows certain reporting guidelines. There are at least two surgical case reporting guidelines now published. The PROCESS Guideline, consisting of an eight item checklist, and the SCARE Guideline, consisting of a 14-item checklist, both developed after a Delphi consensus exercise aim at improving the reporting quality of surgical case reports.
In conclusion, some of the questions I would ask when I am asked to review a case report is:

- Is there a learning point in this case report?

- Can it lead to a change in practice?

- Could it be applied if I get a similar case?

Reports which showcase an unusual complication of a surgical procedure or how to treat an uncommon problem arising in a case would have great value as it would give the reader some new knowledge and confidence. It would also alert them to look for similar findings in their practice.

Always look to see how the case report would benefit the reader facing a similar situation and write it with an aim to disseminating the new knowledge you have gained from dealing with this case so that others benefit. It should not be an exercise in just increasing your publication list.

Publisher's Note Springer Nature remains neutral with regard to jurisdictional claims in published maps and institutional affiliations. 